\title{
Die Interkulturelle Pragmatik - \\ Angenehm, Sie kennenzulernen!
}

\author{
KaTHRIN SIEBOLD \\ Universidad Pablo de Olavide, Sevilla
}

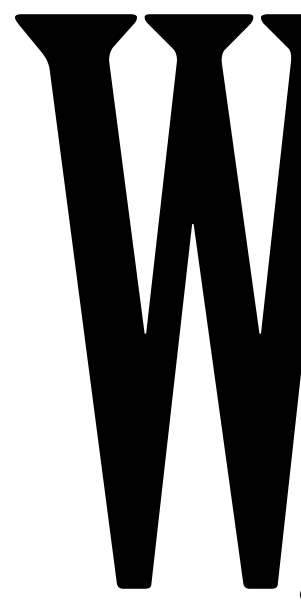

enn Spanier und Deutsche in interkulturelle Kommunikation treten, so kann es vorkommen, dass der Spanier sich darüber wundert, wie unverblümt der Deutsche seine skeptische Meinung in Diskussionen äußert, wie direkt er Missstände kritisiert, wie ehrlich er auf die höfliche Frage qué tal antworten kann, wie ernst er mitunter Einladungen nach Spanien nimmt oder wie übertrieben er sich für die selbstverständlichsten Dinge bedankt. Dem Deutschen hingegen mag vielleicht auffallen, dass ein qué tal von spanischer Seite nicht immer als Gesprächseinstieg gedacht ist, dass der Spanier nicht vor Imperativen in Bitten zurückschreckt, sich bei längeren Schweigephasen in Gesprächen unwohl fühlt, eventuell ein sí, ya veremos einer direkten Absage vorzieht und unbekannte Gegenüber problemlos als hijo, cielo oder reina ansprechen kann.

Wie diese Beispiele verdeutlichen, können spezifische Sprachnormen und Stile verbaler Höflichkeit, die das Interaktionsverhalten innerhalb einer Gemeinschaft prägen, von Kultur zu Kultur Divergenzen aufweisen. Solche kulturspezifischen Kommunikationsmuster werden im Forschungsbereich der Interkulturellen Pragmatik, die in den letzten Jahren zunehmend an Bedeutung gewonnen und zahlreiche interessante Studien (vgl. u.a. Sifianou 1992, Márquez Reiter 2000, Nixdorf 2001, Díaz Pérez 2003, Meyer 2007) hervorgebracht hat, sprachvergleichend analysiert. Während die kontrastive Sprachanalyse im traditionellen Sinn phonologische, syntaktische und semantische Differenzen und Parallelen verschiedener Sprachsysteme unter-

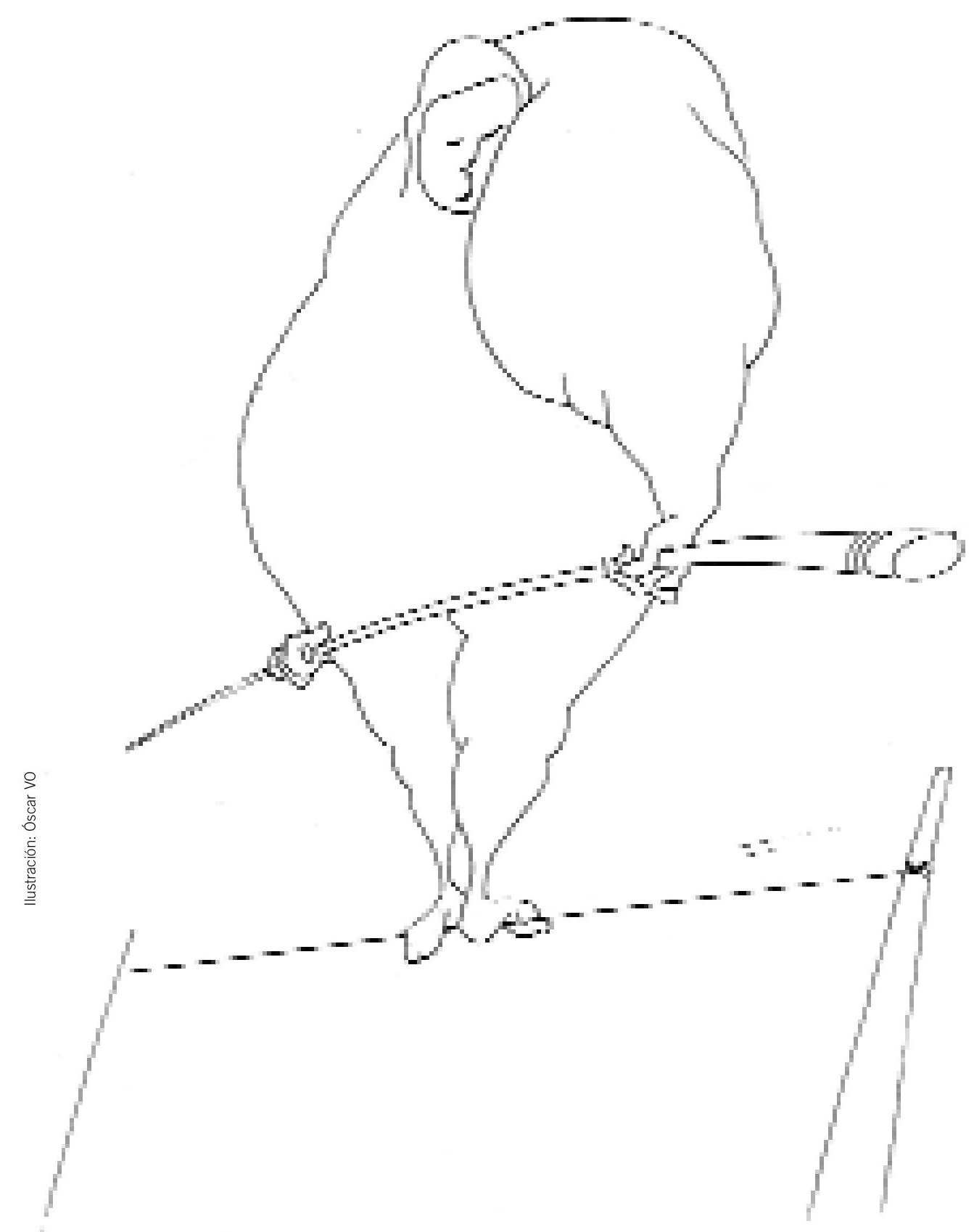


sucht, beschäftigt sich die Interkulturelle Pragmatik mit der kulturspezifischen und kontextabhängigen Sprachverwendung.

Zentraler Forschungsgegenstand ist die kontrastive Analyse der Realisierung bestimmter Sprechakte in verschiedenen Sprechergemeinschaften, wobei das Hauptaugenmerk auf kulturspezifische Direktheits- und Indirektheitsgrade, sprachliche Routinen sowie syntaktische und lexikalische Modifizierung zur Intensivierung oder Abschwächung der Sprechakte gelegt wird. So hat beispielsweise Meyer (2007) herausgefunden, dass es signifikante Unterschiede im Beschwerdeverhalten zwischen deutschen und amerikanischenglischen Muttersprachlern gibt. Letztere vollzögen, wie Meyer (2007: 260) erklärt, ihre Beschwerden in der Regel unter starker Berücksichtung der sozialen Anerkennung des Gesprächspartners als erstere, die direkt und inhaltsorientiert argumentierten.

Weiterhin befasst sich die Interkulturelle Pragmatik mit unterschiedlichen Diskurskonventionen und untersucht hier beispielsweise, durch welche Merkmale sich Gesprächseröffnungen und -beendigungen von Kultur zu Kultur unterscheiden. In diesem Zusammenhang wird des öfteren auf den vergleichsweise geringen Gebrauch von beziehungsorientierten Routineformeln in deutschen Gesprächseröffnungen hingewiesen, während in anderen Kulturen eine länge «Aufwärmphase» vor der Besprechung des eigentlichen Anliegens durchaus zum guten Ton gehört (vgl. Helmolt/Müller 1993: 531). Andere Fragestellungen im Bereich der Diskursorganisation beziehen sich auf kulturspezifische Präferenzen bei der Themenwahl, die Funktion von small talk, die Regulierung des Sprecherwechsels sowie Formen und Funktionen von Diskursmarkern und Hörersignalen.

\section{Pragmatischer Transfer}

Die Interkulturelle Pragmatik geht davon aus, dass Fehlkommunikation und Missverständnisse in der interkulturellen Kommunikation größtenteils auf Interferenzfehlern, d.h. auf einer inadäquaten Übertragung der gewohnten sprachlichen Kommunikationsmuster und Verhaltensweisen von der Muttersprache in die Zielsprache, in einer Situation, in der sie mitunter keine oder nicht dieselbe Gültigkeit haben, beruhen.

Je nach den Konsequenzen der Übertragung wird üblicherweise zwischen negativem und positivem Transfer unterschieden. Führt das Transferergebnis zu einer angemessenen Äußerung in der Zielsprache, handelt es sich um positiven Transfer, während es bei negativem Transfer zu pragmatischen Misserfolgen kommen kann. Weiterhin differenziert man nach Thomas (1983) zwischen pragmalinguistischem und soziopragmatischem Transfer. Pragmalinguistische Transferprozesse implizieren formale und funk- tionale Aspekte des sprachlichen Ausdrucks, soziopragmatischer Transfer hängt dagegen mit den sozialen Kontextbedingungen zusammen (vgl. auch Meyer 2007: 29ff.).

Die Tatsache, dass pragmatischer Transfer häufig auch bei fortgeschrittenen Lernern auftritt, birgt insofern ein großes Konfliktpotential in sich, als pragmatische Unterschiede bei grammatischer Korrektheit nicht immer bewusst wahrgenommen werden. Dies kann zu fundamentalen Attributionsfehlern führen, d.h. Verhaltensauffälligkeiten werden nicht als Resultat kulturspezifischer Kommunikationsmuster identifiziert, sondern allein auf die Persönlichkeit des Gesprächspartners bezogen (vgl. Thomas 1993: 385), was letztlich zu stereotypen Zuweisungen bestimmter Eigenschaften an eine Gesamtkultur führen kann.

\section{Methodische Vorgehensweise}

Das Instrument, das in der Interkulturellen Pragmatik mit Abstand am häufigsten zur Sammlung des Datenmaterials verwendet wird, ist das sogenannte Diskursergänzungsverfahren (Discourse Completion Test $)^{1}$. Hierbei handelt es sich um einen schriftlichen Fragebogen, in dem von den Probanden in einer Art Sandwich-Verfahren ein bestimmter Sprechakt zwischen zwei bestehende Äußerungen geschrieben werden soll. Diese Datenerhebungsmethode gewährleistet aufgrund der möglichen Festlegung kontextueller Variablen die Vergleichbarkeit der Daten und ermöglicht zudem eine unkomplizierte Sammlung großer Datenmengen (vgl. Kasper/Dahl 1991: 46). Meines Erachtens bringt sie jedoch den elementaren Nachteil mit sich, dass sie in ihrer schriftlich überdachten Form keine authentisch gesprochene Sprache reflektiert. Zum einen haben die Versuchspersonen nicht die Möglichkeit, den Sprechakt über mehrere Redesequenzen hinweg interaktiv auszuhandeln, wie es in authentischen Gesprächssituationen der Fall ist. Zum anderen müssen sie nicht spontan mündlich reagieren, sondern überlegen, wie sie in einer bestimmten Situation sprechen würden und das dann verschriftlichen. Wie eine Studie von Wolfson et al. (1989: 182) belegt, gibt es aber oft relevante Unterschiede zwischen der eigenen Sprachwahrnehmung bzw. dem Bewusstsein darüber, wie man zu sprechen meint, und dem tatsächlichen Sprachgebrauch.

Die zweithäufigste Erhebungsmethode innerhalb der Interkulturellen Pragmatik ist das Rollenspiel, in dem zwei Interaktanten eine bestimmte Gesprächssituation vorgelegt bekommen, in der sie dann frei kommunizieren können. Das Rollenspiel eignet sich meiner Ansicht nach besser für die Datensammlung, denn durch die Festlegung des Kontexts kann das Kriterium der Vergleichbarkeit ebenso erfüllt werden wie im Diskursergänzungs- 
verfahren. Darüberhinaus trägt diese Mehtode zu einer gesteigerten Authentizität der verwendeten Sprache bei, weil sie den Probanden spontane mündliche Sprachproduktion abverlangt, ihnen die Entwicklung des Sprechakts über mehrere Redesequenzen hinweg ermöglicht und zudem zusätzliche verbale, paraverbale und nonverbale Informationen (z.B. Mimik, Gestik, Proxemik, Stimmlage, Pausen, Unterbrechungen, Überlappungen, Hörersignale etc.) liefert, die im Diskursergänzungsverfahren nicht erfasst werden können. Hauptnachteil der Rollenspielmethode ist der große Arbeits- und Zeitaufwand, der durch die Organisation der Sprachaufnahmen und deren mühsame und langwierige Transkription entsteht.

\section{Brückenschläge zu anderen Disziplinen}

In der Interkulturellen Pragmatik werden kulturspezifische Kommunikationsmuster nicht nur kontrastiv beschrieben. Durch den Rückgriff auf verschiedene Theorien aus den unterschiedlichsten Wissenschaftsbereichen, die das kommunikative Verhalten der Menschen beleuchten und analysieren, werden auch Erklärungen für die unterschiedlichen Interaktionsstile angeboten bzw. gesucht.

Besonders sinnvoll erweist sich in diesem Zusammenhang der Rekurs auf die Theorien zur Höflichkeit, deren bekannteste von Brown und Levinson (1987) auf dem zentralen Konzept des face beruht. Wie Brown und Levinson darlegen, stellt das face für jeden einzelnen einen Wert dar, der aus zwei unterschiedlichen Bedürfnissen besteht, zum einem das gesellschaftliche Bestätigung und Anerkennung suchende positive face, zum anderen das nach Freiheit strebende negative face, das von anderen nicht bedrängt werden will. Die Hauptfunktion der Höflichkeit besteht nach Brown und Levinson darin, durch die Anwendung verschiedener Höflichkeitsstrategien gesichtsbedrohende Sprechakte ( $\mathrm{fa}$ ce threatening acts) abzuschwächen und so durch die Wahrung des eigenen und des fremden face eine harmonische und reibungslose Kommunikation zu gewährleisten. Bei den möglichen Höflichkeitsstrategien kann mit Brown und Levinson zwischen solchen Strategien unterschieden werden, die sich am positiven $\mathrm{fa}$ ce orientieren und Aufmerksamkeit und Solidarität bekunden und solchen, die sich an das negative face richten und respektvolle Distanz einhalten.

Ein weiteres Forschungsgebiet, das wertvolle Erklärungsansätze zu den Kulturspezifika in der Kommunikation liefert, ist die Kulturanthropologie. Als zentrale Forschungsarbeit aus der kognitiven Kulturanthropologie sei die Erhebung des belgischen Wirtschaftswissenschaftlers Hofstede (1997) genannt, der anhand eines 5-Dimensionen Modells (hohe/geringe Machtdistanz, Individualismus/Kollektivismus, Feminität/Maskulinität, hohe/geringe Unsicherheitsvermeidung und Langzeit-/Kurzzeitorientierung) kulturelle Unterschiede klassifiziert. Bei solcherart Spezifizierungen sollte man nicht aus den Augen verlieren, dass es sich um riskante Abstraktionen handelt und Kultur weder statisch abgeschlossen noch homogen ist, sondern in ihrer Flexibilität ständig dynamischen Prozessen unterliegt und äußerst heterogene Merkmale aufweist (vgl. Heringer 2007). Trotzdem kann ein Basiswissen über kulturspezifische Tendenzen innerhalb der 5 Dimensionen Aufschluss über bestimmte Merkmale im Kommunikationsverhalten geben. Als ein Beispiel seien Hofstedes Erklärungen über das Diskussionsverhalten in kollektivistischen und individualistischen Kulturen genannt. Hofstede legt dar, dass in stärker kollektivistisch ausgeprägten Kulturen eher eine harmonische Konfliktregelung gesucht und meistens indirekt kommuniziert wird, während individualistische Kulturen direkte Auseinandersetzungen nicht scheuen.

Auch der Rückgriff auf die Sozialpsychologie, insbesondere auf die kulturvergleichenden Studien zu den sogenannten Kulturstandards, die in verschiedenen Kulturen wirksam sind, erweist sich für Deutungsversuche der in der Interkulturellen Pragmatik beschriebenen Phänomene als interessant. Kulturstandards sind «diejenigen Werte, Normen, Regeln und Einstellungen in einer Kultur, die sich gerade im zwischenmenschlichen Bereich umfassend auf Wahrnehmung, Denken, Urteilen und Handeln ihrer Mitglieder auswirken» (Markowsky/Thomas 1995: 7) und als spezifische Spielregeln des gesellschaftlichen Lebens in einer Kultur angesehen werden können. Zentrale deutsche Kulturstandards, die in verschiedenen Forschungsprojekten (z.B. Thomas 1991, Markowsky/Thomas 1995) ermittelt wurden, seien u.a. die «Interpersonale Distanz» (Markowsky/Thomas 1995: 33), eine anfängliche Distanziertheit und Verschlossenheit, die nach einer längeren Phase des Kennenlernens einer überraschenden Offenheit und Zugänglichkeit weicht, oder die "Direktheit interpersonaler Kommunikation» (Markowsky/Thomas 1995: 53), die Priorität des Inhaltsaspekts, der offenen Meinungsäußerung und des sachbezogenen Argumentierens vor dem Beziehungsaspekt, und viele andere Kulturstandards wie eine starke Regelorientierung, ein ausgeprägtes Pflichtbewusstsein oder ein großes Organisationsbedürfnis, die hier nicht alle beschrieben werden können. Für die spanische Kultur werden in einer Studie von Dunkel (2004), die für die Zusammenarbeit in internationalen Arbeitsgruppen relevante Kulturstandards identifiziert hat, folgende zentrale Standards ermittelt: Spanier wiesen in der Kommunikation einen starken Personenbezug auf, für sie gelte, «personal relations are much more important than business» (Dunkel 2004: 158). Außerdem manifestierten sie, so Dunkel, eine ausgeprägte Hierarchieorientierung, aus der Folgendes resultiere: «Hierarchical structures and positions have to be respected when communicating, they are sometimes even more important than competences and capabilities» (Dunkel 2004: 169). Weitere zentrale spanische Kulturstandards, die Dunkel vorstellt, wie ein polychrones Zeitkonzept, eine schwache Trennung zwischen Privat- und Arbeitsleben oder ein starkes Autoritätsdenken seien hier nur kurz angesprochen.

Die Interkulturelle Pragmatik erweist sich auch hinsichtlich der Anwendung ihrer Forschungsergebnisse als interdisziplinäre Forschungsrichtung. So können wichtige Erkenntnisse über pragmatische Eigenheiten bestimmter Sprachen nicht nur für den Fremdsprachenunterricht, sondern auch für die Didaktik der Wirtschaftskommunikation und des Übersetzens und Dolmetschens fruchtbar gemacht werden. Diese Art interdisziplinären Arbeitens wird in Spanien beispielsweise von Lucrecia Keim verkörpert, die aus ihren Untersuchungen zur Interkulturellen Pragmatik (vgl. Keim 2000, 2004) stets didaktische Schlüsse zieht und konkrete Aufgabenvorschläge für den Deutschunterricht unterbreitet. Dabei weist sie auf die Wichtigkeit der vernetzten Arbeit auf den Ebenen des soziokulturellen, des pragmalinguistischen und des diskursorganisatorischen Wissens hin und unterstreicht die Bedeutung von Aufgaben, die das situationsgebundene Einsetzen von 
Kommunikationsstrategien (metakommunikativen Redewendungen und Paraphrasierungen) zum Ziel haben.

\section{Spanischdeutsche Einblicke}

Im Bereich der Interkulturellen Pragmatik sind in den letzten Jahren die verschiedensten Sprachenpaare kontrastiv untersucht worden, wobei Englisch als meist analysierte Sprache eine zentrale Stellung einnimmt (vgl. z.B. Sifianou 1992, Márquez Reiter 2000, Nixdorf 2001, Díaz Pérez 2003, Meyer 2007, die alle das Englische mit einer anderen Sprache kontrastieren).

Das Sprachenpaar Spanisch-Deutsch hingegen ist bislang noch vergleichsweise unerforscht. Jürgen Wolff hat mit seinen Arbeiten zur interkulturellen Kommunikation zwischen Spaniern und Deutschen (Martín/Wolff 1983, Wolff 1994 u.a.) sicherlich Pionierarbeit geleistet und wichtige Anstöße zu weiteren Untersuchungen gegeben. Basierend auf Beobachtungen und Erfahrungen beschreibt er Missverständnisse, die aufgrund kulturspezifischer Kommunikationsmuster in Gesprächen zwischen Deutschen und Spaniern auftreten können.

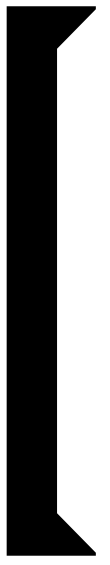

Meiner Ansichtnach ist dieser kulturspeeifische Strategiengebraulch auf den unterschiedlichen Stellenwert des positiven und des negativen face in beiden Kulturen zurüuckzuführen.

Eine der ersten systematischen Untersuchungen im Bereich der deutsch-spanischen Interkulturellen Pragmatik finden wir bei Keim (1994). Die Autorin untersucht auf der Grundlage von Verhandlungssimulationen interkulturelle Interferenzen im Bereich der deutschspanischen Wirtschaftskommunikation. Die Bereiche, die sich dabei als besonders sensibel für interkulturelle Interferenzen herausstellen, sind die Rederechtorganisation und die Imagearbeit. Am deutlichsten können Unterschiede bei der Prozedur der Rederechtübernahme, bei der Verwendung von positiven und negativen Höflichkeitsstrategien und bei den Techniken zur Stützung des face nachgewiesen werden. Bei den spanisch-spanischen Gesprächen entstehen häufig längere Überlappungen, insbesondere in durch hohen Dissens gekennzeichneten Sequenzen, während auf deutscher Seite einer der beiden Partner in der Regel das Rederecht aufgibt. Im Bereich der Imagearbeit fällt auf deutscher Seite eine höhere Tendenz zur Verwendung der negativen Höflichkeit auf.

Bedeutend für die spanisch-deutsche Interkulturelle Pragmatik sind auch die Arbeiten von Josefa Contreras. In ihrer Dissertation (2005) zeigt sie auf, dass es in bestimmten Gesprächssituationen Interventionen mit Überlappungen gibt, die als Höf- lichkeitsstrategien betrachtet werden können. Sie folgt in ihren Deutungen dem face-Konzept von Bravo (1999) und kritisiert den Ethnozentrismus der Höflichkeitstheorie von Brown und Levinson. In einer späteren Analyse der direktiven Sprechakte in spanischen und deutschen Alltagsgesprächen (2007) geht sie über die kontrastive Beschreibung hinaus und analysiert die Hörerreaktionen, um herauszufinden, wie in den jeweiligen Sprechakten die Höflichkeit auf beiden Seiten interpretiert wird.

Eine weitere Studie auf dem Gebiet der spanisch-deutschen Interkulturellen Pragmatik habe ich selbst durchgeführt (Siebold 2008). Als Textkorpus dienen 126 gefilmte und transkribierte Rollenspiele (spanisch-spanisch, deutsch-deutsch) in verschiedenen Alltagssituationen, die den direktiven Sprechakt der Bitte sowie die expressiven Sprechakte der Entschuldigung, des Kompliments und der Komplimenterwiderung hervorrufen. Die kontrastive Analyse der Daten zeigt auf, dass die sprachliche Realisierung aller untersuchten Sprechakte erhebliche kulturspezifische Divergenzen aufweist, die im Folgenden anhand einiger Beispiele kurz zusammengefasst werden sollen.

Bezüglich des Bittverhaltens fällt bei den spanischen Sprechern eine höhere Tendenz zur Verwendung direkter Strategien wie Imperative, aus der Lokution ableitbare Bitten oder direkte Fragen auf, wie folgende Beispiele verdeutlichen.

(1) Niña, dame cinco croissants y una barrita, anda.

(2) Tengo boy un invitado en mi casa y me voy a llevar un poquito de más pan (...), y me va a poner dos croissants, uno de chocolate y otro relleno de crema.

(3) Hola Señora, buenas tardes, mire, busco el Corte Inglés, me han dicho que está en la calle Luis Montoto, ¿está lejos?

Dahingegen bevorzugen die deutschen Probanden, wie aus den Beispielen 4 und 5 hervorgeht, indirektere Strategien wie den Wunschausdruck oder die Frage nach den Vorbereitungsbedingungen, in diesem Fall nach der Fähigkeit des Hörers, die erbetene Handlung auszuführen.

(4) Ich hätte gern fünf Croissants und ein Baguette, bitte.

(5) Ach, entschuldigen Sie bitte. Ich kenne mich hier überhaupt nicht aus und ich möchte so gern zum Marienplatz. Können Sie mir vielleicht mal sagen, wie ich dahin komme?

Meiner Ansicht nach ist dieser kulturspezifische Strategiengebrauch auf den unterschiedlichen Stellenwert des positiven und des negativen face in beiden Kulturen zurückzuführen. Die bevorzugten Strategien auf spanischer Seite stellen einen relativ direkten Eingriff in das persönliche Terrain des Gesprächspartners dar, d.h. dessen negatives face wird durch den Strategiengebrauch nicht geschützt. Auffällig ist jedoch die Anwendung anderer Höflichkeitsmittel, die das positive face des Gegenübers stimulieren, wie die Koseform niña (Beispiel 1), der interpersonelle Marker anda (Beispiel 1) und die Kombination verschiedener Aufmerksamkeitssignale hola señora, buenas tardes, mire (Beispiel 3), die alle zur Schaffung einer freundschaftlichen Atmosphäre und sozialer Nähe zwischen den Gesprächspartnern beitragen und so die Gesichtsbedrohung durch den direkten Gebrauch des Imperativs kompensieren. 
Im Kontrast dazu erfolgt in den deutschen Konversationen durch den Einsatz der indirekten Strategien eine klare Abschwächung der illokutiven Kraft der Bitte mittels negativer Höflichkeitsformen, die das negative face des Interaktanten stärker berücksichtigen. In vielen Fällen wird die Bitte weiterhin durch lexikalische oder syntaktische Modifizierer wie mal, vielleicht (Beispiel 5) oder den Konjunktiv II (Beispiel 5) abgemildert. Der kombinierte Gebrauch dieser verschiedenen Höflichkeitsmittel verdeutlicht, dass die deutschen Sprecher sich in hohem Maße um die Wahrung des negativen face ihrer Gesprächspartner bemühen.

ÄhnlicheSchlussfolgerungen können auch bei der Analyse der spanischen und deutschen Entschuldigungen gezogen werden.

(6) A: ¿Usted no sabe que aquí está prohibido aparcar?

B: Sí, no no, pero es que han sido dos segundos que he dejado abi a mi madre.

(7) A: Sie stehen im Halteverbot. Ich wollte Sie gerade aufschreiben.

B: Ah, das tut mir furchtbar leid. Ich musste dringend zur Post.

Charakteristisch für die sprachliche Realisierung der spanischen Entschuldigungen ist zum einen, dass nicht immer eine explizite Entschuldigung durch performative Verben ausgedrückt wird, sondern in vielen Gesprächen indirekte Strategien wie Erklärungen (Beispiel 6) als Entschuldigungen fungieren. Zum anderen wird die illokutive Kraft vieler spanischer Entschuldigungen durch das Herunterspielen der Fehlhandlung abgeschwächt, wie die Äußerung es que han sido dos segundos in Beispiel 6 zeigt.

Im Vergleich dazu verwendet die Mehrheit der deutschen Muttersprachler eine explizite Entschuldigungsformel, die häufig durch lexikalische Modifizierer wie furchtbar in Beispiel 7 intensiviert und durch den Gebrauch indirekter Strategien wie Erklärungen noch unterstützt wird.

Bedenkt man, dass Entschuldigungen durch das Eingeständnis von Fehlverhalten eine starke Bedrohung für das positive $f a-$ ce des Sprechers, also den Wunsch nach gesellschaflticher Anerkennung und Achtung, darstellen, wird durch die eher indirekten und zusätzlich abgeschwächten Entschuldigungen auf spanischer Seite wieder die Tendenz zum Schutz des positiven face des Sprechers deutlich, während auf deutscher Seite anhand der expliziten und teilweise noch verstärkten Entschuldigungen weni-

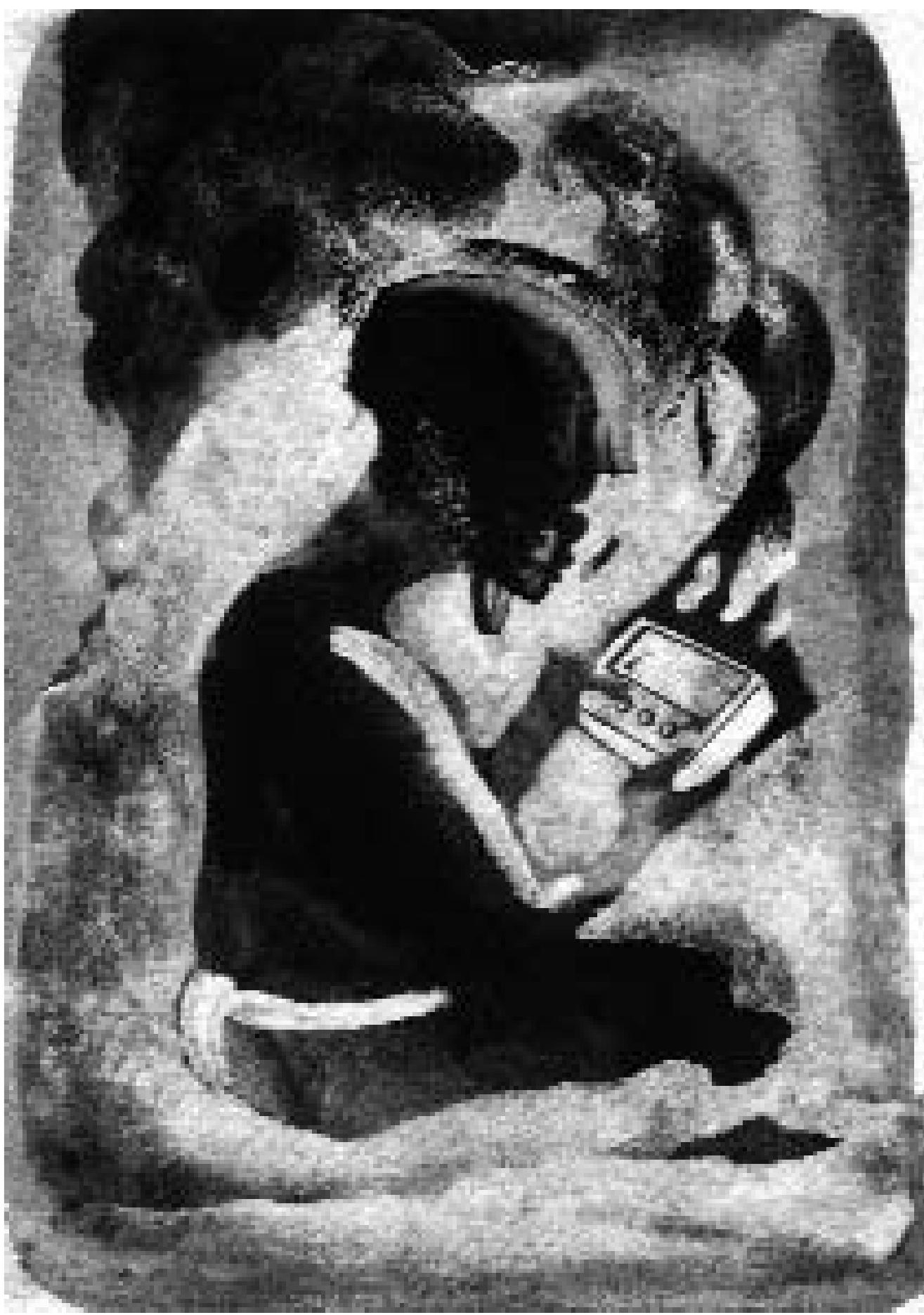

ger Rücksicht darauf genommen wird.

Als letztes Beispiel für pragmatische Unterschiede im spanischen und im deutschen Kommunikationsverhalten seien die Kulturspezifika in den Komplimenterwiderungen erwähnt. Bei den Komplimenterwiderungen stehen den Sprechern verschiedene Strategien zur Verfügung, die sich zwischen den beiden Polen der Annahme und der Ablehnung des Kompliments befinden.

\section{(7) A: ¡Qué barbaridad! ¡Qué guapa vienes! \\ B: Gracias.}


(8) A: ¡La que veo muy bien es a usted!

B: Sí, hijo, no está mal.

(9) A: Du hast aber einen tollen Pullover an! Wo hast du denn den her?

B: Findest du? Na ja, den hat mir meine Frau gekauft. So schön finde ich den gar nicht.

(10)A: Mensch, der ist ja echt schön! Der steht dir auch total super!

B: Den habe ich bei Wenz gekauft. Soll ich dir mal einen Katalog mitbringen?

Die klar präferierten spanischen Strategien sind die Annahme der Komplimente durch Dankausdruck (Beispiel 7) bzw. durch Zustimmung (Beispiel 8), während die Komplimente in den deutschen Gesprächen häufiger umgeleitet werden, indem der Sprecher den Komplimentgegenstand anzweifelt (Beispiel 9) oder sich in Erklärungen über dessen Ursprung bzw. Anschaffung ergeht (Beispiel 9 und 10).

Auch die kulturspezifische Realisierung dieses Sprechakts lässt sich nach dem gleichen Deutungsmuster wie die vorhergehenden

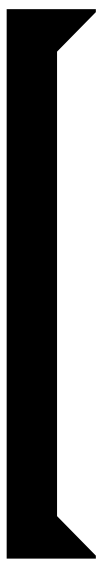

Die illokutive Kraft vieler spanischer Entschul-

digungen wird durch das Herunterspielen der

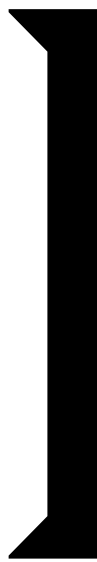

erklären, nämlich mit dem unterschiedlichen Stellenwert der positiven Höflichkeit in beiden Kulturen. Das Kompliment als klassisches Instrument zum Ausdruck positiver Höflichkeit wertet das face des Gesprächspartners auf. Es nicht anzunehmen, bedeutet nicht nur eine Gesichtsbedrohung für den Sprecher selbst, sondern auch den Ausdruck von Nicht-Zustimmung mit dem Gesprächspartner, was dessen positives face verletzen könnte. Die spanisch-deutsche Interkulturelle Pragmatik stellt ein sehr interessantes und fruchtbares Forschungsgebiet dar, das noch lange nicht abgegrast ist. Es bleibt zu hoffen, dass in zukünftigen Studien kulturspezifische Kommunikationsstile in bislang nicht untersuchten Sprechakten und Diskurskonventionen herausgearbeitet werden, die sich nach und nach zu einem Gesamtbild der pragmatischen Eigenheiten beider Sprachen zusammenfügen.

Für Anregungen und Kommentare bin ich sehr dankbar (ksiex@upo.es)

1. Dieses Verfahren wurde erstmals im wegweisenden Pionierprojekt CCSARP (Cross Cultural Speech Act Realization Projekt) von Blum-Kulka et al. (1989) angewendet und ist seitdem von zahlreichen Forschern im Bereich der Interkulturellen Pragmatik übernommen worden.

\section{Auswahlbibliographie}

Blum-Kulka, Shoshanaetal. (1989): Cross-cultural pragmatics: requests and apologies. Norwood.

Bravo, Diana (1999): «Imagen positiva vs. Imagen negativa: pragmática sociocultural y componentes de face", ORALIA 2, 155-184.

Brown, Penelope/Levinson, Stephen (1987): Politeness. Some universals in language usage. Cambrigde.

ConterasFernández,Josefa(2005): El uso de la cortesía y las sobreposiciones en las conversaciones. Un análisis contrastivo alemán-español. Valencia.

ContrerasFernández,Josefa (2006): «Imagen social española versus imagen social alemana», in: Blas Arroyo et al. (eds.): Discurso y sociedad: contribuciones al estudio de la lengua en contexto social, 605-612.

Contreras Fernández, Josefa (2007) «Cortesía lingüística en actos directivos", Pragmática, Discurso y Sociedad. Quaderns de Filologia. Estudis Lingüistics. Vol. XII 9-14, 175-191.

Díaz Pérez, Francisco (2003): La cortesía verbal en inglés y en español: actos de habla y pragmática intercultural. Jaén.

Dunkel, Amanda (2004): «Culture Standards and their impact on teamwork-An empirical análisis of Austrian, German, Hungarian and Spanish culture differences", Journal for East European Management Studies 9, 147-174.

Helmolt, Katharina von/Müller, Bernd-Dietrich (1993): «ZurVermittlung interkultureller Kompetenzen", in: Müller, BerndDietrich (ed.): Interkulturelle Wirtschaftskommunikation. München.

Heringer, Hans Jürgen (2007): Interkulturelle Kommunikation. Tübingen.

Hofstede, Geert. (1997): Lokales Denken, globales Handeln, Kulturen, Zusammenarbeitund Management. München.

Kasper, Gabriele/Dahl, Merete (1991): «Research methods in interlanguage pragmatics", Studies in Second Language Adquisition 13, 215-247.

Keim, Lucrecia (1994): Interkulturelle Interferenzen in der deutsch-spanischen Wirtschaftskommunikation. Frankfurt.

Keim, Lucrecia (2000) «Interkulturelle Verständigung in der Wirtschaftskommunikation", in: Ehnert, Rolf(ed.): Wirtschaftskommunikation kontrastiv. Frankfurt, 189-208

Keim,Lucrecia (2004): «Interkulturalität und Interaktion im FSU mit Übersetzungsstudierenden", in: Fleischmann, Eberhard et al. (eds.): Translationskompetenz. Tübingen, 609622.

Markowski, Richard/Thomas, Alexander (1995): Studienhalber in Deutschland. Interkulturelles Orientierungstraining für amerikanische Studenten, Schülerund Praktikanten. Heidelberg.

Márquez Reiter, Rosina (2000): Linguistic politeness in Britain and Uruguay. A contrastive stu$d y$ of requests and apologies. Amsterdam.

Meyer, Katrin (2007): Interkulturelle Pragmatik: Aufforderungen, Entschuldigungen und Beschwerden. Hamburg.

Nixdorf, Nina (2001): Höflichkeit im Englischen, Deutschen, Russischen. Ein interkultureller Vergleich am Beispiel von Ablehnungen und Komplimenterwiderungen. Marburg.

Siebold, Kathrin (2008): Actos de habla y cortesía verbal. Estudio pragmalingüistico e intercultural. Frankfurt.

Sifianou, Maria (1992): Politeness phenomena in England and Greece. A cross cultural perspective. Oxford.

Thomas, Alexander (1993): Kulturvergleichende Psychologie. Göttingen

Thomas, Jenny (1983): «Cross cultural pragmatic failure», $A p$ plied Linguistics 4, 91-112.

Torres, Gracia M./Wolff, Jürgen (1983): «Interkulturelle Kommunikationsprobleme beim Sprachenlernen.-dargestellt an Mißverständnissen zwischen Spaniern und Deutschen.», Neusprachliche Mitteilungen aus Wissenschaft und Praxis, 209-216.

Wolfson, Nessa (1989): «Problems in the comparison of speech acts across cultures», in: BlumKulka et. al (eds.), 174-196. 